\title{
Design Considerations on a Resistive Superconducting Fault Current Limiter
}

\author{
J. KOZAK*, M. MAJKA AND S. KOZAK \\ Lublin University of Technology, Faculty of Electrical Engineering and Computer Science, \\ Department of Electrical Engineering and Electrotechnologies, \\ Nadbystrzycka 38A, 20-618 Lublin, Poland \\ Doi: 10.12693/APhysPolA.138.710 \\ *e-mail: j.kozak@pollub.pl
}

\begin{abstract}
Recent changes in power networks such as a stronger need for interconnection and the rise of distributed generation result in an increasing demand for protection against high fault currents. Superconducting fault current limiters provide a solution for handling such faults. The application of a superconducting fault current limiter in a power network leads to a reduction in a hazard potential and savings in the switchgear equipment. This paper presents the design of a conduction cooled $6 \mathrm{kV}$ superconducting fault current limiter.
\end{abstract}

topics: superconducting fault current limiter, resistive SFCL, superconductivity

\section{Introduction}

Because of the development of the energy industry, the wind power industry in particular, electricity networks — designed to manage electricity provided by large system power stations - are not capable of absorbing such a large excess of generated power which threatens the development of the renewable energy sector. Connecting new power generating units to the network must be safe for the entire network infrastructure and at the same time it must secure the required parameters of energy quality. When a new energy source is connected to the network, there is an increase of shortcircuit power at the connection point. This means that during a short-circuit, the value of a fault current in such a network increases. The increased fault current poses a threat to the technical infrastructure of the network due to the dynamic and thermal effects of the passage of the fault current. Substantial network investments are required to solve this problem, thus we should seek new methods of increasing the connection capacity of the existing networks.

In fact, even if the permission is obtained to connect new power generating installation, a modernization or construction of an entire new section of the power network would be required. High-power superconducting AC installations, such as superconducting fault current limiters, facilitate a better utilization of the already existing power network infrastructure. Such installations increase the connection capacity for renewable sources, improving the quality of electric power and have an environmental impact of reducing $\mathrm{CO}_{2}$ emission.
The restriction of thermal and dynamic effects of the passage of the fault current can be obtained by limiting the short-circuit time and the value of the maximum initial fault current. Possibilities to shorten the time of the short-circuit offered by automatic systems are limited.

In the case of conventional technology, there is often no adequate method of effectively detecting and limiting a short circuit. The application of current limiting reactors only adds the unwanted inductance and impedance to the grid, leading to real and reactive power losses as well as to potential voltage instabilities. Limiters based on pyrotechnical means require external triggering, direct access to the device and an expensive exchange of parts after each limiting event. During operation, the superconductors of the limiter are cooled to a temperature of around $-200^{\circ} \mathrm{C}$ which is accomplished by a single stage helium cryocooler. At nominal power loads, the superconductors have no electrical resistance at the cryogenic temperatures. If the critical current of a superconductor is exceeded, the material suddenly loses its superconducting properties and starts to behave like a normal conductor. This resistance limits the current to a predefined value. These material properties only make superconductors ideal self-actuating current limiting elements [1-10].

\section{Operation principle and behavior in the grid}

The superconductor has a nonlinear voltagecurrent characteristic $V \sim\left(I / I_{c}\right)^{n}$. Here, the superconductor critical current $I_{c}$ is defined as the current, at which a $1 \mu \mathrm{V}$ voltage drop per cm superconductor length is reached. 


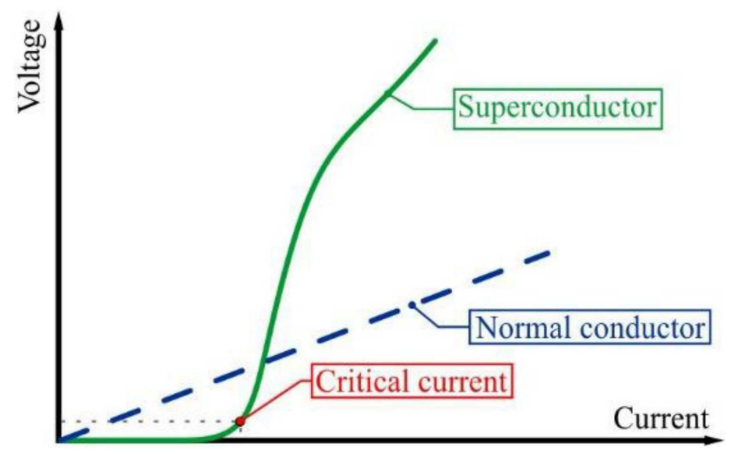

Fig. 1. Voltage-current characteristics of a superconductor compared to a normal conductor.

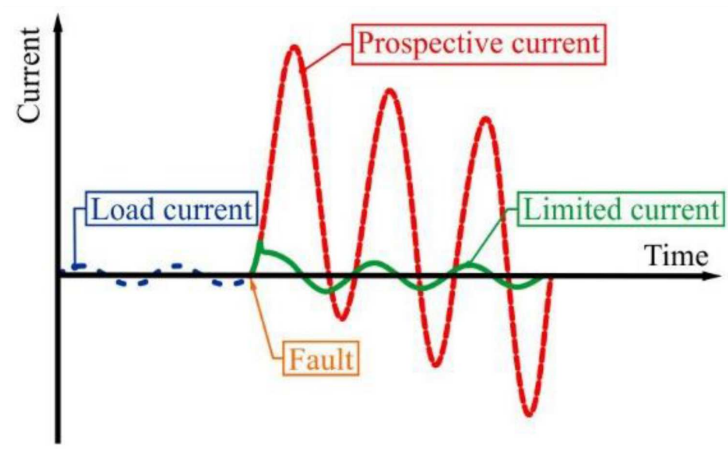

Fig. 2. Prospective and limited current during a short-circuit.

For the typical high temperature superconductors, the exponent $n$ is usually between 5 and 30 . During the short circuit, the current increases and the voltage follows this power law. Within a few milliseconds, the related heat moves the superconductor above its critical temperature. Then, the material loses the superconducting properties and behaves like a normal resistor with a proportional law (Fig. 1).

A continued heating of the superconductor during the short circuit results in further resistance growing and limiting the current (Fig. 2). The transition from the superconducting to the normal conducting state is called a quench. In the superconducting fault current limiter (SFCL), when the normal operating current is below the critical current $I_{c}$, the quench is triggered by the high current which occurs during a short circuit event [4]. Superconducting fault current limiters have unique characteristics of almost zero impedance under normal operating conditions and high impedance at fault conditions. The SFCL technology makes it possible to design a low impedance grid structure, for instance by low impedance equipment or grid coupling without the risk associated with high short circuit power [5].

During a short circuit, a resistive SFCL in the line reduces the phase shift between the current and voltage and thus also the stress and requirements regarding the circuit breakers in the line. Because of that, the current and the voltage are almost in phase. Circuit breakers, buses and cables downstream of the limiter can have much lower ratings and a considerable equipment cost may be saved. Moreover, owing to the use of SFCLs, the equipment can be operated close to its limits and, consequently, investment can be avoided or postponed until a later date by installing a SFCL [6-10].

\section{SFCL design}

\subsection{Superconducting tape selection}

A SF12100 (12 mm width) tape has been selected for constructing the SFCL. The tape with no copper stabilizer and with thicker substrate $(100 \mu \mathrm{m})$ heats up slower than SF1250 (50 $\mu \mathrm{m}$ substrate) and will be used in a fault current limiter to ensure longer thermal withstand times. The characteristics presented in Fig. 3 allow us to determine the temperature to which a superconductor tape SF12100 will be heated at the moment of turning off a fault current, with a predetermined voltage value for $1 \mathrm{~m}$ of the HTS tape $\left(U_{1 \mathrm{~m}}\right)$. An increase of voltage per length unit of the HTS tape causes an increase of the superconducting tape's temperature. The switching off time by a circuit breaker connected in a series with SFCL is less than $80 \mathrm{~ms}$. The temperature of the HTS tape should not exceed $300 \mathrm{~K}$ (calculated for $U_{1 \mathrm{~m}}=52.5 \mathrm{~V}$ ) which is still far from maximum acceptable temperature $T_{\max }=450 \mathrm{~K}$.

\subsection{Coil design}

The SFCL windings have the same length as the HTS tape wound in opposite directions and connected in parallel. The parameters are detailed in Table I. The presented design assures a resistive character of the limiter (see Fig. 4) and also reduces internal forces during the passage of the current.

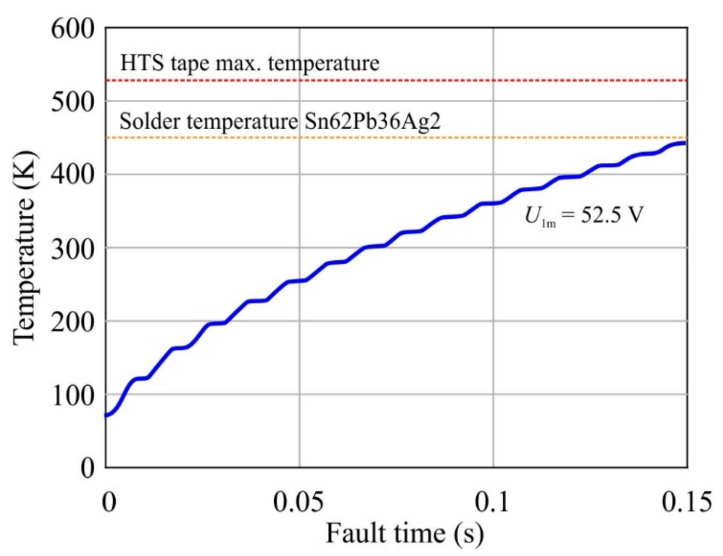

Fig. 3. Temperature increase for SF12100 superconducting tape model during the fault current for voltage value per $1 \mathrm{~m} \mathrm{[10].}$ 
TABLE I

Parameters of SFCL windings $(2 \times$ SF12100 HTS tapes)

\begin{tabular}{l|c|c}
\hline \hline \multicolumn{2}{c|}{ Parameters description } & Value \\
\hline nominal voltage & $U_{N}$ & $6 \mathrm{kV}$ \\
nominal current & $I_{N}$ & $140 \mathrm{~A}$ \\
overload current & $I_{\text {overload }}$ & $420 \mathrm{~A}$ \\
critical current $(2 \times 300 \mathrm{~A})$ & $I_{\mathrm{CSFCL}}$ & $600 \mathrm{~A}$ \\
$I_{\text {RMS@ IC }}$ & $I$ & $424 \mathrm{kA}$ \\
voltage per 1 m tape length & $U_{1 \mathrm{~m}}$ & $52.5 \mathrm{~V}$ \\
length of HTS tape per winding & $l$ & $66 \mathrm{~m}$ \\
length of single turn & $l_{\text {turn }}$ & $2.31 \mathrm{~m}$ \\
winding height & $h$ & $0.44 \mathrm{~m}$ \\
winding diameter & $d$ & $0.736 \mathrm{~m}$ \\
number of turns & $n_{\text {turn }}$ & 28.5
\end{tabular}

TABLE II

AC losses in HTS coils $(2 \times 66 \mathrm{~m}$ of SF12100 HTS tapes)

\begin{tabular}{c|c|c}
\hline \hline AC losses & Current [A] & AC losses [W] \\
\hline $0.5 I_{N}$ & 70 & 0.02 \\
$I_{N}$ & 140 & 0.2 \\
$I_{\text {overload }}$ & 420 & 13.2 \\
& & \\
&
\end{tabular}

Fig. 4. Schematic of HTS coils.

The superconducting tape's length (given in meters) equals

$$
l=\frac{U_{N}}{\sqrt{3} U_{1 \mathrm{~m}}}=66 .
$$

Losses in superconducting winding depend on the frequency of the alternating current and the value of the current [11]. Table II presents the $\mathrm{AC}$ losses calculated for the superconducting coils of SFCL.

\subsection{Current leads}

The project assumes the contact method of cooling the limiter and current leads. Due to the limited power of the cryocooler, the heat flow through the current leads and the Joule losses generated at the flow of the rated and overload current should be limited. The copper part of

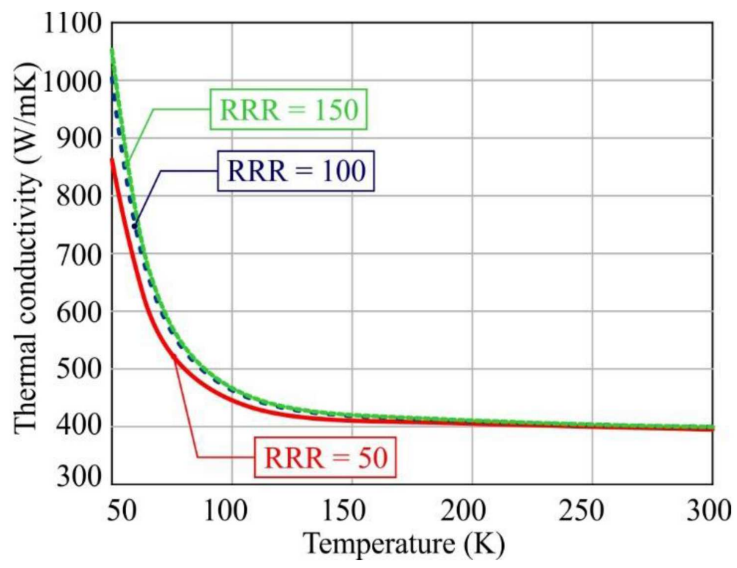

Fig. 5. Copper thermal conductivity versus temperature [12].

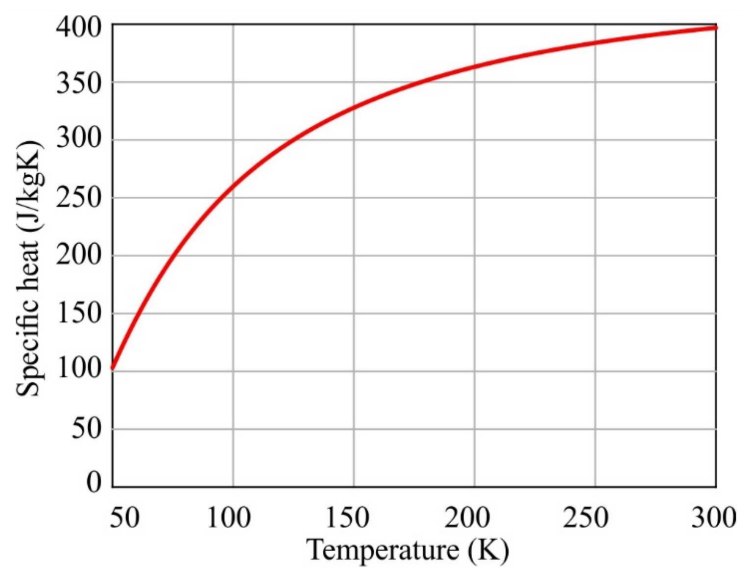

Fig. 6. Copper specific heat vs. temperature [12].

the limiter's current leads consists of a part at room temperature and a part with a specific crosssectional area and a length whose purpose is to limit the heat entering the limiter. The thermal power generated in the cryostat (mainly by the current leads) should be less than the cooling capacity of the cryocooler at $65 \mathrm{~K}$. Assuming room temperature (upper part of the current lead) $T_{H}=293 \mathrm{~K}$, coils temperature (lower part of the current lead) $T_{L}=65 \mathrm{~K}$, cryocooler cooling capacity $Q_{L}=115 \mathrm{~W}$ obtained at $65 \mathrm{~K}$, nominal current of SFCL $I_{N}=140 \mathrm{~A}$, SFCL overload current $I_{\text {overload }}=3 I_{N}=420 \mathrm{~A}$, current of the current lead $I_{\mathrm{CL}}=1.45 I_{\text {overload }}=609 \mathrm{~A}$ (safety coefficient), current lead length $L=150 \mathrm{~mm}$ and current lead thickness $b=2 \mathrm{~mm}$, the width of the current lead $a$ should be calculated. Both the thermal conductivity coefficient (Fig. 5) and the copper specific heat (Fig. 6) change with temperature [12]. The change in copper thermal conductivity as a function of temperature for various residual resistivity ratio (RRR) values is shown in Fig. 5. In the temperature range from 65 to $300 \mathrm{~K}$, the copper thermal conductivity coefficient for different $R R R$ values reaches 


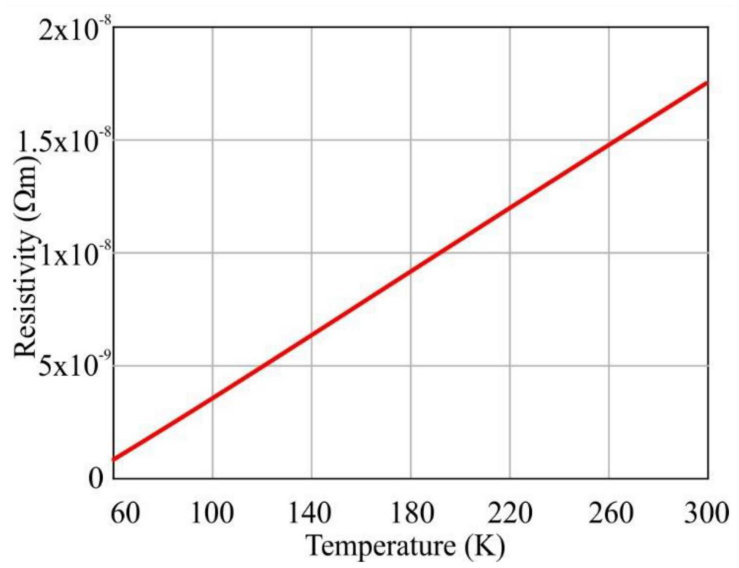

Fig. 7. Copper resistivity vs. temperature [12].

Heat loss per 1 current lead TABLE III

\begin{tabular}{c|c}
\hline \hline Current $[\mathrm{A}]$ & Heat loss $[\mathrm{W}]$ \\
\hline 0 & 13.1 \\
140 & 13.8 \\
420 & 19.35 \\
609 & 26.2
\end{tabular}

similar values (Fig. 5), so copper with a coefficient $\mathrm{RRR}=50$ can be used to make the current leads. Copper resistivity increases with temperature (Fig. 7), so the cross-section of copper leads can be decreased with a decrease in temperature while maintaining the same thermal power generated in the copper leads. The optimal value of the current depends on the $A$ - current lead cross-section, the $L$ - length of the current lead [13], and $k(T)$ - the thermal conductivity. This dependence is expressed by

$$
I_{\mathrm{opt}}=\frac{A}{L} \int_{T_{L}}^{T_{H}} \frac{k(T)}{\sqrt{2 \int_{T}^{T_{H}} k(T) \rho(T) \mathrm{d} T}} \mathrm{~d} T .
$$

Based on the analysis of the results in Fig. 8, the width of the current lead was selected for the project to be $a=10 \mathrm{~mm}(L / A=7500)$.

\subsection{Cooling}

The cryocooler operating point for the rated current is below the cooling capacity curve (Fig. 9). The cryocooler head has been selected to provide a surplus cooling power when operating with the overload current. For the overcurrent, the losses in the cryostat increase approximately twofold. Both superconducting windings of the limiter have 285 turns of the superconducting tape SF12100. Each winding has $66 \mathrm{~m}$ of the HTS tape wound in opposite directions and connected in parallel. Windings have a fiberglass structure and insulation. The terminals of the windings are situated on the internal side of the windings structure and are connected to the current leads.

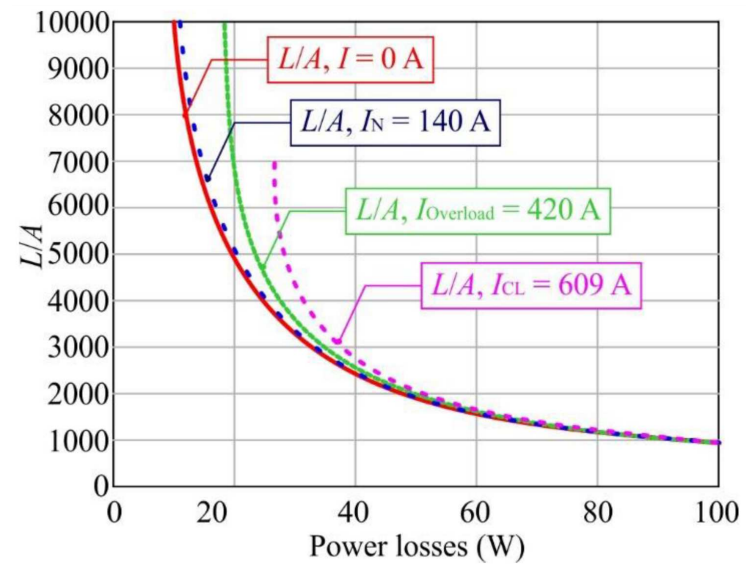

Fig. 8. The $L / A$ ratio vs. power losses of the current leads for selected current values.

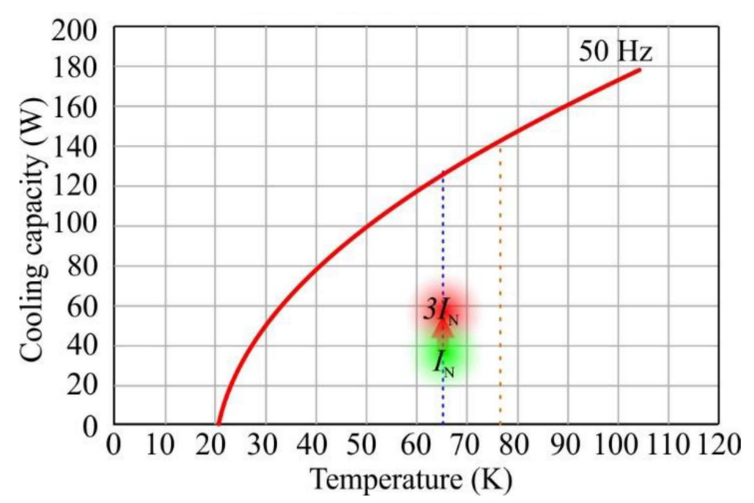

Fig. 9. Cryocooler head work point for the rated and overload current.

Additional multilayer insulation (MLI) covering the windings reduces the heat transfer from the cryostat walls to the windings. The currents leads and the superconducting windings are cooled by a single stage cryocooler. Ceramic insulators of the current leads assure good thermal transfer and electrical insulation (Fig. 10). The overall losses in the cryostat at $65 \mathrm{~K}$ are calculated below $40 \mathrm{~W}$, taking into account the current lead losses (Table III, thermal conduction of the structure support as well as radiation. The SFCL parameters are presented in Table I and the design is shown in Fig. 11. The applied solution of conduction cooled SFCL with compensated and parallel connected superconducting windings substantially limited the voltage in the limiter in nominal conditions. The resistive character of the limiter - due to the design of the compensated windings - minimizes the electromotive forces inside the cryostat during a fault. There are no coil interconnections that add the unwanted resistance inside the cooled coils except for two soldered joints between coils ends and current leads. Such a solution makes the limiter simple in structure, reliable and easy to manufacture. The fiberglass structure of the superconducting windings is light and nonconductive 


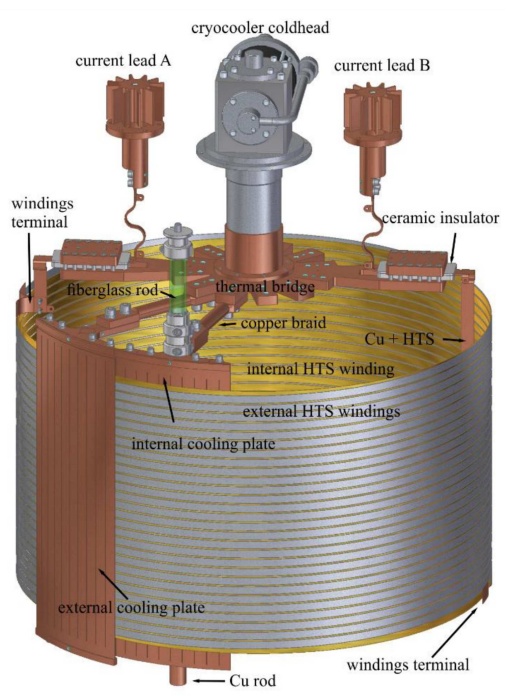

Fig. 10. HTS windings construction of SFCL.

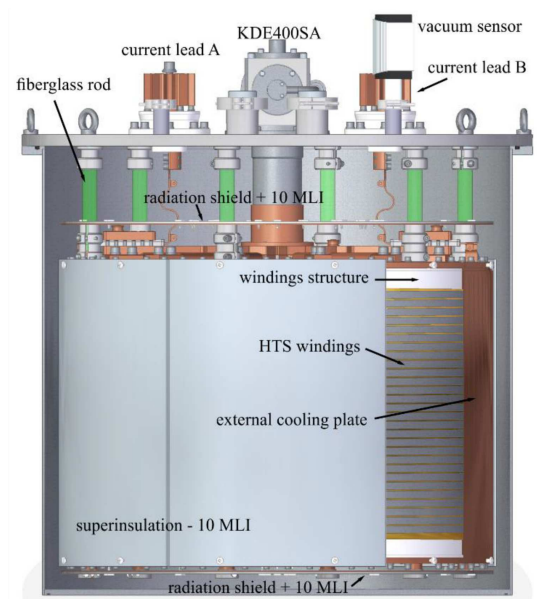

Fig. 11. The SFCL cross-section.

of electrical current. The copper plates incised into vertical stripes ensure good conductive cooling of the windings and reduce the eddy currents' losses. The presented design leads to the simple and compact superconducting fault current limiter.

\section{Conclusion}

We have successfully designed and tested a conduction cooled $6 \mathrm{kV}$ resistive superconducting fault current limiter which consists of two compensated and parallel connected superconducting windings.

\section{Acknowledgments}

This work was supported in part by the National Fund for Environmental Protection and Water Management and the National Centre for Research and Development under Grant GEKON2/O2/267193/13/2015.

\section{References}

[1] A. Morandi, Physica C 484, 242 (2013).

[2] J. Bock, "Short-Circuit Protection to a Fault: Superconducting Fault Current Limiters", in: Power Engineering International, no. 6, 2010.

[3] J. Kozak, M. Majka, S. Kozak, T. Janowski, IEEE Trans. Appl. Supercond. 23, 5600604 (2013).

[4] J. Bock, in: Proc. 21st Int. Conf. on Electricity Distribution, 2011, p. 0352.

[5] A. Hobl, in: CIRED Workshop, 2012, p. 0296.

[6] M. Majka, S. Kozak, Przeglad Elektrotechniczny 85, 183 (2009).

[7] M. Majka, J. Kozak, S. Kozak, G. Wojtasiewicz, T. Janowski, IEEE Trans. Appl. Supercond. 25, 5601005 (2015).

[8] D. Czerwinski, L. Jaroszynski, T. Janowski, M. Majka, J. Kozak, IEEE Trans. Appl. Supercond. 24, 5600104 (2014).

[9] O. Naeckel, M. Noe, IEEE Trans. Appl. Supercond. 24, 5601605 (2014).

[10] M. Majka, J. Kozak, S. Kozak, IEEE Trans. Appl. Supercond. 27, 5601405 (2017).

[11] B. Shen, J. Li, J. Geng, et al., Physica $C$ 541, 40 (2017).

[12] N.J. Simon, E.S. Drexler, R.P. Reed, NIST Monograph, Vol. 177, U.S. Department of Commerce, National Institute of Standards and Technology, 1992.

[13] H.-M. Chang, M.J. Kim, Cryogenics 49, 210 (2009). 